\title{
Integration of Results from Time-Resolved Serial Crystallography and Spectroscopy in the Catalysis of Ceftriaxone by Beta-Lactamase
}

\author{
Jose L. Olmos, Jr. ${ }^{a}$, Hector A. Chaires ${ }^{a}$, Mitchell D. Miller ${ }^{a}$, George N. Phillips, Jr. ${ }^{a, b}$ \\ aDepartment of BioSciences, Rice University, Houston, TX, 77005, USA, olmos@rice.edu \\ ${ }^{b}$ Department of Chemistry, Rice University, Houston, TX, 77005, USA
}

The visualization of protein motions is one of the many exciting, cutting-edge uses of an X-ray free electron laser. Time-resolved mix-and-inject-serial (MISC) crystallography studies have directly visualized the reaction catalyzed by the enzyme, $\beta$-lactamase (Olmos et al.). This class of enzymes confers antibiotic resistance by hydrolyzing a broad spectrum of antibiotics in many bacteria, including Mycobacterium tuberculosis. The previous MISC results using the thirdgeneration antibiotic, ceftriaxone, showed the development of an intermediate and the approach to a steady state involving product inhibition. While this approach has directly shown the course of the reaction in near-atomic detail, the technique has some practical limitations stemming from the packing of molecules in the crystal, the need for high concentrations of substrate, and the lack of synchrony throughout the crystal. Nevertheless, kinetic behavior can be determined from the structural information, including the postulation of product inhibition, which was subsequently confirmed by Szyperski, et al. (unpublished). ${ }^{2}$

To further connect the crystallography result to enzymology, the enzymatic reaction was studied in solution under conditions that more closely mimic the reaction in the microcrystals. In particular, UV-vis spectroscopy was employed to monitor the activity under various conditions. The solution reaction rates are indeed variable and dependent upon the components of the solution used for crystallization. Compared to the standard conditions for assays, $\mathrm{pH}$ and other buffer dependent effects were observed on the enzymatic activity.

Taken together, the integrated results of MISC and UV-vis spectroscopy show new insights for the basic enzymological activity of a medically important enzyme.

References

[1] Olmos, et al. BMC Biology 2018 16:59 https://doi.org/10.1186/s12915-018-0524-5

[2] Szyperski BioXFEL $6^{\text {th }}$ International Meeting, Febuary 2019, San Diego, CA.

https://www.bioxfel.org/resources/1670/download/Speaker_Abstracts_2019.pdf

Acknowledgements: This work is supported by the NSF Graduate Research Fellowship Program under Grant No. R3E821 (to JLO) and the NSF STC BioXFEL Center Award No. 1231306. 